\title{
Management of common bile duct stones with a biliary endoprosthesis. Report on 40 cases
}

\author{
R Peters, P Macmathuna, M Lombard, J Karani, D Westaby
}

\begin{abstract}
Endoscopic placement of a biliary endoprosthesis has been proposed for the management of choledocholithiasis when stone extraction is difficult or considered hazardous. Over a two year period this approach was used in $\mathbf{4 0}$ such patients. There were 24 women and 16 men with a median age of 76 years. In seven patients with severe cholangitis no attempt was made to extract the stones. Twenty three (57.5\%) patients underwent a sphincterotomy and four $(10 \%)$ needle knife papillotomy. The endoprosthesis insertion was considered a temporary measure in $13(32.5 \%)$ patients and definitive treatment in $27(67 \cdot 5 \%)$. Bile duct drainage was established in all patients. Early complications occurred in six patients (15\%), but were without sequelae. Late complications developed in eight $(20 \%)$ of the patients and included biliary colic (four), cholangitis (three), and cholecystitis (one). Two patients (one cholangitis and one cholecystitis) died as a consequence of the complication. Only patients without a sphincterotomy developed cholangitis. A total of eight patients $(20 \%)$ underwent surgery (one as an emergency) and nine a repeat endoscopic retrograde cholangio pancreatography (two as an emergency) to clear the duct. The remaining 23 patients are asymptomatic at a median of 13 months (range five to 24 months). Biliary endoprosthesis insertion for choledocholithiasis is an important alternative means of establishing drainage in selected cases, and is probably the optimum method of management for the elderly and or debilitated patients with previous cholecystectomy. Caution must be exercised, however, in patients with an in situ gall bladder.

(Gut 1992; 33: 1412-1415)
\end{abstract}

Choledocolithiasis is a relatively frequent problem arising in approximately $15 \%$ of patients with stones in the gall bladder. ${ }^{\prime}$ Before the availability of endoscopic bile duct intervention surgical treatment was the usual approach to management. The mortality of exploring a common bile duct dramatically increases over the age of 60 particularly if other serious medical conditions are present such as diabetes, malnutrition, cardiorespiratory and renal insufficiency.' In one study the mortality ranged between 2.5 and $33 \%$ in patients over 70 years depending on the surgical approach used. ${ }^{2}$ Endoscopic sphincterotomy is now the primary treatment for bile duct stones in most clinical contexts, whether emergency or elective. Surgical treatment for bile duct stones is now restricted to those patients who are not obstructed and require a cholecystectomy, and in any patient in whom endoscopic treatment fails to achieve bile duct clearance. ${ }^{3}$

Endoscopic sphincterotomy and basket extraction of the stones has been the most widely used technique having an overall success rate of $85 \%$ in clearing the ducts. In patients in whom the procedure is successful $4 \cdot 4-9 \cdot 8 \%$ will develop bile duct stenosis, new stone formation or both. The major limitations of therapy have been the presence of large stones $(>15-20 \mathrm{~mm}$ ), large and tortuous ducts, non-dilated ducts in which the basket cannot expand, and a stricture distal to the stone $(\mathrm{s}){ }^{4}$ Other techniques have been developed to enhance bile duct clearance including mechanical lithotripsy, electrohydraulic lithotripsy, chemical dissolution using methylterbutylether through a biliary catheter, extracorporeal shockwave lithotripsy and laser fragmentation. ${ }^{5}$

The use of a biliary endoprosthesis has been proposed as an alternative approach for patients at high risk for surgery. ${ }^{3}$ A number of small series have been published providing support for the efficacy of this technique. ${ }^{6-14}$ The role played by an endoprosthesis is believed to be two-fold. First, prompt biliary drainage is established in the obstructed duct and second, subsequent impactation of stones is prevented by the presence of the endoprosthesis within the duct. Endoprosthesis occlusion (which is almost inevitable within three to six months) will not influence this function and therefore replacement is not routinely required. A second group in whom this technique may be valuable is those in whom the number of stones or technical difficulties such as diverticuli, may expose the patient to a protracted procedure which even if successful may be detrimental for the patient that is, the old and frail. We report our experience in 40 patients in whom such criteria were fulfilled and in whom this technique was applied.

\section{Methods}

\section{PATIENTS}

From January 1989 to January 1991 a total of 146 patients underwent endoscopic retrograde cholangiopancreatography for choledocholithiasis. In 106 of these patients $(72 \cdot 6 \%)$ the stones were successfully retrieved by the standard technique of sphincterotomy and basket or balloon extraction. In the remaining 40 patients $(27 \cdot 3 \%)$ clearance of the ducts was not achieved because of the aberrant position of the papilla (seven), large size of the stones (17), distorted common bile duct (four), presence of a biliary stricture distal to the stones (five) or in the presence of serious clinical conditions in which endoprosthe- 
TABLE I Clinical presentation

\begin{tabular}{lrl}
\hline & $(n)$ & $\%$ \\
\hline RUQ pain & 32 & 80 \\
Jaundice & 31 & $77 \cdot 5$ \\
Fever & 12 & 30 \\
Sepsis & 7 & $17 \cdot 5$ \\
Abnormal LFT's & 2 & 5 \\
Pancreatitis & 2 & 5 \\
\hline
\end{tabular}

Note: some patients with more than one abnormality. RUQ= right upper quadrant; LFT=liver function test. sis insertion was considered the most rapid means of establishing drainage (seven). Patient demographic, clinical and laboratory data are summarised in Table I, II, and III. There were 24 female and 16 male patients with a median age of 76 (range 24-92). All patients underwent an abdominal ultrasound examination before endoscopic retrograde cholangiopancreatography. This confirmed intra and extrahepatic duct dilatation in 31 patients $(77 \%)$, cholecystolithiasis in 19 of the 32 patients $(60 \%)$ in whom the gall bladder was in place. Common bile duct stones were only shown in 10 patients $(25 \%)$.

The endoscopic retrograde cholangiopancreatography was carried out in the standard manner using an Olympus or Pentax sideviewing endoscope under antibiotic cover. An attempt was made to extract the bile duct stones in 33 $(82.5 \%)$ of the patients. In seven cases severe cholangitis was considered a specific indication for the rapid establishment of drainage using an endoprosthesis. After the initial cannulation and passage of a guide wire, a $7 \mathrm{~F}$ double pigtail polyethylene endoproshesis was placed with the proximal end above the stones and the distal end in the duodenum. A straight $10 \mathrm{~F}$ endoprosthesis was used in the five patients with a bile duct stricture distal to the stones.

\section{Results}

The preliminary endoscopy confirmed a periampullary diverticulum present in 14 (35\%) patients. A median of two stones were found (range one to six) within the duct with a median size of $1.5 \mathrm{~cm}$ (range $0 \cdot 5-2 \cdot 5$ ). A stone was impacted at the level of the ampulla in five patients. In five patients a common bile duct stricture was found, four benign (believed to be as a consequence of choledocholithiasis in two and as a complication of cholecystectomy in the other two). One stricture was subsequently proven to be malignant (metastatic carcinoid).

TABLE II Laboratory features

\begin{tabular}{|c|c|c|c|c|c|}
\hline & $(n)$ & Abnormal & $\%$ & $\begin{array}{l}\text { Value } \\
(\text { median })^{\star}\end{array}$ & Range \\
\hline $\begin{array}{l}\text { ALP } \\
\text { BIL } \\
\text { GGT } \\
\text { AST }\end{array}$ & $\begin{array}{l}40 \\
40 \\
29 \\
40\end{array}$ & $\begin{array}{l}40 \\
31 \\
27 \\
13\end{array}$ & $\begin{array}{l}100 \\
77 \cdot 5 \\
93 \\
32 \cdot 5\end{array}$ & $\begin{array}{l}326 \mathrm{IU} / 1 \\
75 \mu \mathrm{mol} / 1 \\
234 \mathrm{IU} / 1 \\
173 \mathrm{IU} / \mathrm{l}\end{array}$ & $\begin{array}{l}100-1573 \\
30-258 \\
110-1174 \\
65-730\end{array}$ \\
\hline \multicolumn{6}{|c|}{$\begin{array}{l}(\mathrm{n})=\text { number of patients in which the assays were performed } \\
\star M e d i a n \text { values of abnormal results. } \\
\text { Note: some patients with more than one abnormality. } \\
\text { ALP=alkaline phosphatase; BIL = bilirubin; GGT=gamma } \\
\text { glutamyl transferase; } \text { AST = aspartate aminotransferase. }\end{array}$} \\
\hline \multicolumn{6}{|c|}{ TABLE III Past medical and surgical history } \\
\hline & & & & $(n)$ & $\%$ \\
\hline \multicolumn{6}{|c|}{ Medical } \\
\hline \multirow{2}{*}{\multicolumn{4}{|c|}{$\begin{array}{l}\text { Cardiovascular disease } \\
\text { Respiratory disease }\end{array}$}} & 8 & 20 \\
\hline & & & & 2 & 5 \\
\hline & 2 & 5 \\
\hline \multicolumn{4}{|c|}{ Malignancy } & 2 & 5 \\
\hline \multicolumn{4}{|c|}{ Pregnancy } & 1 & $2 \cdot 5$ \\
\hline \multicolumn{4}{|c|}{ Morbid obesity } & 1 & $2 \cdot 5$ \\
\hline \multicolumn{4}{|c|}{ Crohn's disease } & 1 & $2 \cdot 5$ \\
\hline \multicolumn{4}{|c|}{$\begin{array}{l}\text { Recurrent pancreatitis } \\
\text { Surgical }\end{array}$} & 1 & $2 \cdot 5$ \\
\hline \multicolumn{6}{|c|}{ Surgical } \\
\hline \multicolumn{4}{|c|}{ Cholecystectomy } & 8 & 20 \\
\hline \multicolumn{4}{|c|}{ Billroth II gastrectomy } & 5 & $12 \cdot 5$ \\
\hline \multicolumn{4}{|c|}{ ERCP \& bile duct clearance } & 2 & 5 \\
\hline
\end{tabular}

Note: some patients with more than one medical condition present.
A sphincterotomy was performed in 23 $(57 \cdot 5 \%)$ of the 40 patients although this was only possible in nine $(64 \%)$ of those with a diverticulum. In four $(10 \%)$ patients a precut papillotomy using a needle knife was carried out to gain access to the common bile duct.

In $13(32.5 \%)$ patients the insertion of the endoprosthesis was considered a temporary measure either before planned surgery ( 10 cases) or a repeat attempt to clear the duct endoscopically (three cases). Of these patients six underwent surgery at a median of 1.25 months (range 0.5-7) from the procedure, four are awaiting surgery at a median of nine months (range four to 14), and three underwent endoscopic clearance of their ducts at a median of one month (range $0 \cdot 5-2$ ). In the remaining 27 patients endoprosthesis insertion was considered definitive treatment.

Bile duct drainage was established in all 40 patients (100\%) after endoprosthesis insertion.

Early complications were defined as those occurring during or within the first 72 hours after the procedure and were present in six patients (15\%). One patient developed a transitory respiratory arrest and the procedure was deferred for the next 48 hours. One patient developed a mild allergic reaction to the contrast medium requiring treatment with $\mathrm{H}_{1}$ blocking drugs. Evidence of sphincterotomy related bleeding occurred in three $(7 \cdot 5 \%)$ patients, although none required blood transfusion. The remaining patient developed fever, right upper quadrant pain and deranged liver function tests with negative blood cultures immediately after the procedure requiring intravenous antibiotics for one week with complete resolution of the episode. This patient had not undergone a sphincterotomy before the insertion of the endoprosthesis.

Late complications were defined as those occurring after 72 hours after the insertion of the endoprosthesis. These were present in eight $(20 \%)$ patients. In three patients cholangitis occurred from two to seven months after the placement of the endoprosthesis. One patient was managed endoscopically with stone extraction and another by cholecystectomy and duct exploration. The remaining patient, a 60 year old man with severe respiratory and left ventricular failure died from sepsis. These three patients were notable for not having undergone a sphincterotomy before the insertion of the endoprosthesis. Four patients $(12.5 \%$ of those with a gall bladder in situ) developed typical biliary colic. Two of these underwent a cholecystectomy and exploration of the bile duct 0.25 and two months respectively from the procedure. The other two patients are awaiting surgery seven and 12 months after endoprosthesis insertion. Finally, an 80 year old man with a history of myocardial infarction, rheumatoid arthritis, and metastatic prostatic carcinoma presented with abdominal pain four months after the procedure. An abdominal radiograph failed to show the endoprosthesis, its absence confirmed at endoscopic retrograde cholangiography which also showed a $1 \mathrm{~cm}$ stone within the duct (presumably migrated from the gall bladder) which was successfully extracted. Two weeks later the 
TABLE IV Complications

\begin{tabular}{llll}
\hline Complications & $(n)(n: 40)$ & $\%$ & Deaths \\
\hline Early & & & \\
$\quad$ Haemorrhage & 3 & $7 \cdot 5$ & 0 \\
Allergic reaction & 1 & $2 \cdot 5$ & 0 \\
Respiratory arrest & 1 & $2 \cdot 5$ & 0 \\
Cholangitis & 1 & $2 \cdot 5$ & 0 \\
$\quad$ Total & 6 & 15 & 0 \\
Late & 4 & 10 & 0 \\
$\quad$ Biliary colic & 3 & $7 \cdot 5$ & 1 \\
Cholangitis & 1 & $2 \cdot 5$ & 1 \\
Cholecystitis & 8 & 20 & 2 \\
$\quad$ Total & & & \\
\hline
\end{tabular}

TABLE V Literature review

\begin{tabular}{lllrl}
\hline Reference & $\begin{array}{l}\text { Patients } \\
(\boldsymbol{n})\end{array}$ & $\begin{array}{l}\text { Biliary } \\
\text { symptoms }\end{array}$ & $\%$ & $\begin{array}{l}\text { Follow up } \\
\text { (median) }\end{array}$ \\
\hline$(6)$ & 22 & 0 & 0 & $16 \cdot 3$ \\
$(7)$ & 22 & 4 & 18 & 14 \\
$(8)$ & 8 & 0 & 0 & 17 \\
$(9) \dagger$ & 43 & $?$ & $?$ & $?$ \\
$(10)$ & 17 & 2 & 12 & 39 \\
$(11) \ddagger$ & 41 & 6 & 15 & $15 \cdot 9$ \\
$(12)$ & 26 & 6 & 23 & 26 \\
$(13)$ & 15 & 5 & 33 & 25 \\
$(14) \#$ & 46 & $?$ & 7 & 31 \\
\hline
\end{tabular}

^Follow up in months; $\uparrow$ No absolute figures given; $†$ Follow up includes patients with complications; \# No absolute figures given In the rest of the reports follow up only of the asymptomatic patients, excluding those who died of unrelated causes, underwent surgery or were treated with a repeat ERCP.

patient became septic with associated jaundice. An ultrasound scan revealed non-dilated ducts and suggested acute cholecystitis. He underwent a partial cholecystectomy but died shortly afterwards as a consequence of sepsis. Early and late complications are summarised in Table IV.

Eight $(20 \%)$ of the 40 patients underwent surgery, six having a cholecystectomy and exploration of the common bile duct and two a cholecystectomy alone (the endoprosthesis being left in situ). This procedure was elective in seven patients and an emergency in one. The median time from endoprosthesis insertion to surgery was two months (range $0 \cdot 25-6$ ). Nine patients (12.5\%) underwent a second endoscopic retrograde cholangiopancreatography for duct clearance which was successful in five, seven were done as an elective procedure and two as an emergency, at a median of two months (range $0 \cdot 25-7)$.

The remaining patients (23) have remained asymptomatic with respect to the biliary system or have died of unrelated causes at a median of 13 months (range 5-24). Of these four have been on a surgical waiting list for a median of 9.5 months (range five to 14).

The total number of deaths was six patients (15\%), two with biliary related sepsis, two of a cerebrovascular accident, and two of disseminated malignancy.

Laboratory data in the 23 asymptomatic patients was normal except in 8 (29\%) which had a mildly raised alkaline phosphatase (mean 172 IU/l), five of these also had a mildly raised gamma glutamyl transferase (mean $156 \mathrm{IU} / \mathrm{l})$.

\section{Discussion}

Few reports have been published on the use of biliary endoprosthesis for the management of common bile duct stones. In 1984 Siegel reported 22 cases with long term stenting $(>3$ months), two patients had the proximal end of the pigtail placed accidentally within the gall bladder, all remained asymptomatic for a mean of 16.3 months. ${ }^{6}$ In the same year Forbes described 22 patients with contraindications for surgery in which an endoprosthesis was placed, of these four (18\%) developed cholangitis, three had a repeat endoscopic retrograde cholangiopancreatography with clearance of the ducts, and two died of unrelated causes, the remaining patients were followed up for a mean of 14 months with no complications. ${ }^{7}$ In 1987 a report from Belgium stated that eight elderly patients were treated in this fashion with no complications arising in a mean follow up of 17 months with one biliary unrelated death. ${ }^{8}$ In an editorial of the same journal van der Heyde mentions 43 patients treated similarly in Holland but gives no further information. ${ }^{9}$ Cotton also presented in 198717 patients, two (12\%) developed biliary related problems requring surgery, one having a stormy postoperative period but no deaths; two had a second endoscopic retrograde cholangiopancreatography with duct clearance and five died of unrelated causes; the remaining eight patients were followed for a mean of 39 months. ${ }^{10}$ In 1988 Cairns reported in abstract form 90 patients treated with an endoprosthesis, in 39 it was a short term measure as they underwent surgery or endoscopic clearance of the duct in a mean of $2 \cdot 4$ months, two (5\%) of these patients developed cholangitis. The other 43 patients underwent long term endoprosthesis placement and were followed up for a mean of 15.9 months; six (15\%) developed cholangitis requiring surgery or endoscopic clearance of the ducts, there were no biliary related deaths." Soomers in 1990 described 26 patients in whom this approach was used, six (23\%) patients developed late procedure related complications five requiring surgery and one replacment of the endoprosthesis, these included duodenal perforation in one, endoprosthesis clogging in two and stent migration in two, the patient that underwent a second endoscopic retrograde cholangiopancreatography also had endoprosthesis clogging. Ten patients died of unrelated causes and the remaining 10 were followed up for a mean of 26 months with no biliary symptoms. ${ }^{12}$ Dufeck, also in 1990 described his experience with the procedure in 15 patients, five (33\%) of whom developed biliary complications, two requiring surgery, and three a repeat endoscopic retrograde cholangiopancreatography to establish drainage; 13 patients were symptom free at a median follow up of 25 months. ${ }^{13}$ Finally, Rao in 1991 reported in abstract form a series of 27 and 19 patients followed for three and two years respectively, $7 \%$ of which developed cholestasis. ${ }^{1+}$ Literature review findings are summarised in Table $\mathrm{V}$.

In the present series we report our experience with biliary endoprosthesis placing for the management of common bile duct stones over a two year period.

Of the 146 patients referred for endoscopic treatment of choledocholithiasis the overall success rate for clearing the common bile duct stones was $72 \cdot 6 \%$ which is somewhat lower than other reported series but might reflect the selection of patients to a specialised centre. 
A periampullary diverticulum was found in 14 (35\%) of the patients. This contrasts markedly with only four $(3.7 \%)$ such cases in the 106 patients managed by sphincterotomy and stone extraction. The presence of a diverticulum frequently adds to the technical difficulties of sphincterotomy and stone extraction with an enhanced risk of duodenal perforation, facts which undoubtedly influenced the greater use of an endoprosthesis in such patients.

The insertion of an endoprosthesis was successful in establishing adequate biliary drainage in all the patients. Early complications were few and without sequelae. Late complications were of much greater concern and arose in eight patients $(20 \%)$ at a median of 3.3 months (range $0 \cdot 25-12$ ) from the initial insertion. Four patients (12.5\% suffered biliary colic, three developed cholangitis, and one acute cholecystitis. The development of cholangitis as a late complication occurred only in patients in whom a sphincterotomy was not carried out and this might be considered a prerequisite in all patients in whom an endoprosthesis is inserted as long term therapy. There were two $(5 \%)$ biliary related deaths, one patient with cholangitis and one with cholecystitis. All of the patients in the late complication group had an in situ gall bladder. Currently, approximately $50 \%$ of all patients undergoing a sphincterotomy for bile duct stones have their gall bladder in situ, and of these 6-20\% subsequently require a cholecystectomy for biliary pain or cholecystitis during a follow up of one to six years. ${ }^{21}$ The incidence of biliary related symptoms of $15 \%$ in the present series is similar to that reported in patients undergoing a sphincterotomy and stone extraction alone with the gall bladder left in situ. ${ }^{15}$ In patients in whom endoprosthesis was inserted as a short term measure before surgery or a further attempt at endoscopic duct clearance the only complication observed with biliary colic in patients with gall bladders in situ (four cases). Thus this approach may be considered both safe and effective as a temporary measure to relieve stone related biliary obstruction.

In conclusion biliary endoprosthesis insertion for common bile duct stones offers an important alternative means of establishing duct drainage in selected cases. In patients without a gall bladder the biliary related complications have been negligible and probably for the elderly and or debilitated patients, this represents the optimum method of management. In patients with an in situ gall bladder the risks of biliary related problems are higher although they are in keeping with the general incidence in patients who are treated with a sphincterotomy and successful duct clearance. Some caution must be exercised, however, in these patients and the advent of laparoscopic cholecystectomy may markedly increase the proportion of such patients considered fit for gall bladder removal.

1 Way L, Sleisenger M. Biliary obstruction, cholangitis and choledocholithiasis. In: Sleisinger M, Fordtran J. Gastrointestinal diseases. 4th ed. Philadelphia: WB Saunders, 1989: 1714-28.

2 Vellacott K, Powell P. Exploration of the common bile duct: a comparative study. Brf Surg 1979; 66: 389-91.

3 Cotton PB, Williams C. Practical gastrointestinal endoscopy, 3rd

ed. Oxford: Blackwell, 1990: 118-56.
4 Cotton PB. Endoscopic management of bile duct stones; (apples and oranges). Gut 1984; 25: 587-97.

5 Classen M, Hagenmuller F, Knyrim K, Frimberger E. Giant bile duct stones - Non surgical treatment. Endoscopy 1988; 20: 21-6.

6 Siegel J, Yatto R. Biliary endoprosthesis for the management of retained common bile duct stones. Am $\mathcal{F}$ Gastroenterol 1984; 79: $50-4$

7 Forbes A, Leung J, Cotton PB. Endoscopic biliary endoprosthesis for large bile duct stones. Gut 1984; 25: A1 186-7.

8 Van Steenbergen W, Pelemans W, Ponette E, Fevery J. Endoscopic biliary endoprosthesis as definitive treatment of elderly patients with large bile duct stones. Neth f Med 1987; 30: 107-16.

9 Van der Heyde M, Tytgat G. Biliary endoprosthesis in gallstone disease. Neth F Med 1987; 30: 105-6.

10 Cotton PB, Forbes A, Leung J, Dineen L. Endoscopic stenting for the long term treatment of large bile duct stones: 2 to 5 year follow up. Gastrointest Endosc 1987; 33: 411-2.

11 Cairns S, Dias L, Salmon P, Cotton PB. Management of failed endoscopic clearance of the common bile duct. Gastroenterology 1988; 94: A57.

12 Soomers A, Nagengast F, Yap S. Endoscopic placement of biliary endoprosthesis in patients with endoscopically unextractable common bile duct stones. Endoscopy 1990; 22: $24-6$.

13 Dufek V, Benes J, Chmel J, Kordac V. Biliary endoprosthesis in the treatment of large common bile duct stones: 2 to 5 year follow up. Endoscopy 1990; 22: 240 .

14 Rao K, Varghese M, Blake H, Theodossi A. Endoscopic biliary stenting in a district general hospital. Gut 1991; 32: A586-7.

15 Escourrou J, Cordova J, Lazorthes F, Frexionos J, Ribet A. Early and late complications after endoscopic sphincterotomy for biliary lithiasis with and without the gall bladder 'in situ'. Gut 1984; 25: 598-602. 